\title{
Optical sensing and determination of complex reflection coefficients of plasmonic structures using transmission interferometric plasmonic sensor
}

\author{
Takumi Sannomiya, ${ }^{1,2, a)}$ Tobias E. Balmer, ${ }^{2,3}$ Christian Hafner, ${ }^{2,4}$ Manfred Heuberger, ${ }^{5}$ \\ and Janos Vörös ${ }^{1,2}$ \\ ${ }_{1}^{1}$ Department of Information Technology and Electrical Engineering, Laboratory of Biosensors \\ and Bioelectronics, ETH Zurich, Zurich 8092, Switzerland \\ ${ }^{2}$ Materials Research Center, ETH Zurich, Zurich 8093, Switzerland \\ ${ }^{3}$ Department of Materials, Laboratory for Surface Science and Technology, ETH Zurich, Zurich 8093, \\ Switzerland \\ ${ }^{4}$ Department of Information Technology and Electrical Engineering, Laboratory for Electromagnetic Fields \\ and Microwave Electronics, ETH Zurich, Zurich 8092, Switzerland \\ ${ }^{5}$ Laboratory for Advanced Fibers, Empa, Swiss Federal Laboratories for Materials Testing and Research, \\ Lerchenfeldstrasse 5, St. Gallen 9014, Switzerland
}

(Received 7 October 2009; accepted 31 March 2010; published online 6 May 2010)

\begin{abstract}
The combination of interferometry and plasmonic structure, which consists of gold nanoparticle layer, sputter coated silicon oxide spacer layer, and aluminum mirror layer, was studied in transmission mode for biosensing and refractive index sensing applications. Because of the interferometric nature of the system, the information of the reflection amplitude and phase of the plasmonic layer can be deduced from one spectrum. The modulation amplitude in the transmission spectrum, caused by the interference between the plasmonic particle layer and the mirror layer, increases upon the refractive index increase around the plasmonic particles due to their coherent backscattering property. Our proposed evaluation method requires only two light sources with different wavelengths for a stable self-referenced signal, which can be easily and precisely tuned by a transparent spacer layer thickness. Unlike the standard localized surface plasmon sensors, where a sharp resonance peak is essential, a broad band plasmon resonance is accepted in this method. This leads to large fabrication tolerance of the plasmonic structures. We investigated bulk and adsorption layer sensitivities both experimentally and by simulation. The highest sensitivity wavelength corresponded to the resonance of the plasmonic particles, but useful signals are produced in a much broader spectral range. Analysis of a single transmission spectrum allowed us to access the wavelength-dependent complex reflection coefficient of the plasmonic particle layer, which confirmed the reflection amplitude increase in the plasmonic particle layer upon molecular adsorption. (C) 2010 American Institute of Physics. [doi:10.1063/1.3405912]
\end{abstract}

\section{INTRODUCTION}

Fabry-Perot type interferometric techniques have been used for more than a century to investigate the thickness and refractive index of thin films. A spacer layer is used to enable the detection of film thickness changes at the nanometer scale using visible light. Biosensing, such as measuring protein adsorption, was also performed by an interferometric approach already in $1942 .{ }^{1}$ In the late 1990 s porous structures for the spacing layer were found to give high sensitivity. ${ }^{2}$ More recently a simple yet sensitive transmission interferometric adsorption sensor (TInAS) was developed, where transmission spectra of a sensor chip consisting of a mirror layer and a spacer layer were monitored. ${ }^{3}$ This technique can also be applied for imaging when combined with an optical correlation filter and a microscope. ${ }^{3}$

Nanoplasmonic biosensing [or localized surface plasmon

\footnotetext{
${ }^{\text {a) }}$ Author to whom correspondence should be addressed. Electronic mail: sannomiya@biomed.ee.ethz.ch.
}

resonance (LSPR)] is another optical sensing technique, which is drawing great attention due to its simplicity and sensitivity. ${ }^{4,5}$ Numerous studies have been already carried out regarding the sensitivity and the optical property of different nanostructures, as the optical response depends on many parameters such as material, structure, and interstructural coupling. Kim et al. ${ }^{6,7}$ have recently performed biosensing by a combination of these two techniques. Their very high sensitivity seems to rely on the porous underlying structure similar to the study of Lin $e t$ al. $^{2}$ A simpler system with solid spacer film in reflection mode has been tested by Hiep et al. ${ }^{8}$, where the achieved signal to noise ratio $(\mathrm{S} / \mathrm{N})$ was not as good as standard LSPR techniques. However, at present there is still a lack of theoretical understanding of such combinations of interferometric and plasmonic techniques, which is probably due to the fact that analytical solutions are limited to very simple cases. An appropriate numerical approach is therefore essential to conduct simulations on nanoplasmonics. The multiple multipole program (MMP) is one of the few reliable simulation methods which can achieve the 
accuracy required for assessing the small resonance change in plasmonic structures upon molecular adsorption. ${ }^{9}$ The combination of interferometry and plasmonic particles requires fine discretization at the plasmonic nanostructures. Furthermore, the large spacer layer must be included in the simulation. MMP is well suited for such problems as it is a boundary discretization technique whereas domain discretization techniques such as finite difference time domain would consume huge memory space.

While most transmission based optical techniques only detect the changes in the intensity, interferometry can access the complex optical property of the system. An external interferometer was already applied to nanoplasmonics to determine the dispersive complex optical property of the plasmonic particles. 10,11 In the case of the "built-in" interferometer, such as a TInAS sensor chip with mirror layer and plasmonic structures on top, it should also be possible to obtain the dispersive complex optical property of the nanoplasmonic layer.

The present study shows that such combination of LSPR sensing and interferometry can be used as optical sensor with equivalent sensitivity to standard LSPR sensing, while the complex reflectivity of the plasmonic structure can also be deduced from a single spectrum. In the first part of this paper the transmission interferometric plasmonic sensor (TIPS) is investigated using the instrumental setup of TInAS (Refs. 3 and 12) and MMP simulations. TIPS is an extended version of TInAS where a plasmonic structure is added on the spacer layer. We prefer to use transmission mode as it has less disturbance from the reflection of the surroundings resulting in easier handling. To extract the signal efficiently, we proposed effective data evaluation methods suited for such interferometric plasmonic sensing technique and evaluated the sensitivity to bulk medium change and protein layer adsorption. In the second part, we calculated the complex optical parameters of the plasmonic structures, or the complex reflection coefficient of the plasmonic particle layer in our system, by carefully analyzing the interferometric pattern of the spectrum. The mechanism of the signal generation of the TIPS system was further investigated from the reflection amplitude change by molecular adsorption. The methodology described in this section can theoretically be applied to standard TInAS system.

\section{METHOD}

\section{A. Experimental}

A TInAS sensor chip with a $25 \mathrm{~nm}$ aluminum mirror layer and a $3 \mu \mathrm{m}$ amorphouslike silicon dioxide layer coated by magnetron sputtering was used for the sensing measurement. ${ }^{3} 50 \mathrm{~nm}$ gold colloid particles (British Biocell) were immobilized on the chip which had been previously coated by poly-(ethyleneimine) for electrostatic binding. ${ }^{13}$ The molecular adsorption measurements were conducted in a flow cell, using a buffer solution consisting of $10 \mathrm{mM} 4-(2-$ hydroxyethyl)piperazine-1-ethanesulfonicacid (HEPES) with $150 \mathrm{mM} \mathrm{NaCl}$, adjusted to $\mathrm{pH}$ 7.4. The concentration of the bovine serum albumin (BSA) for the protein adsorption mea-

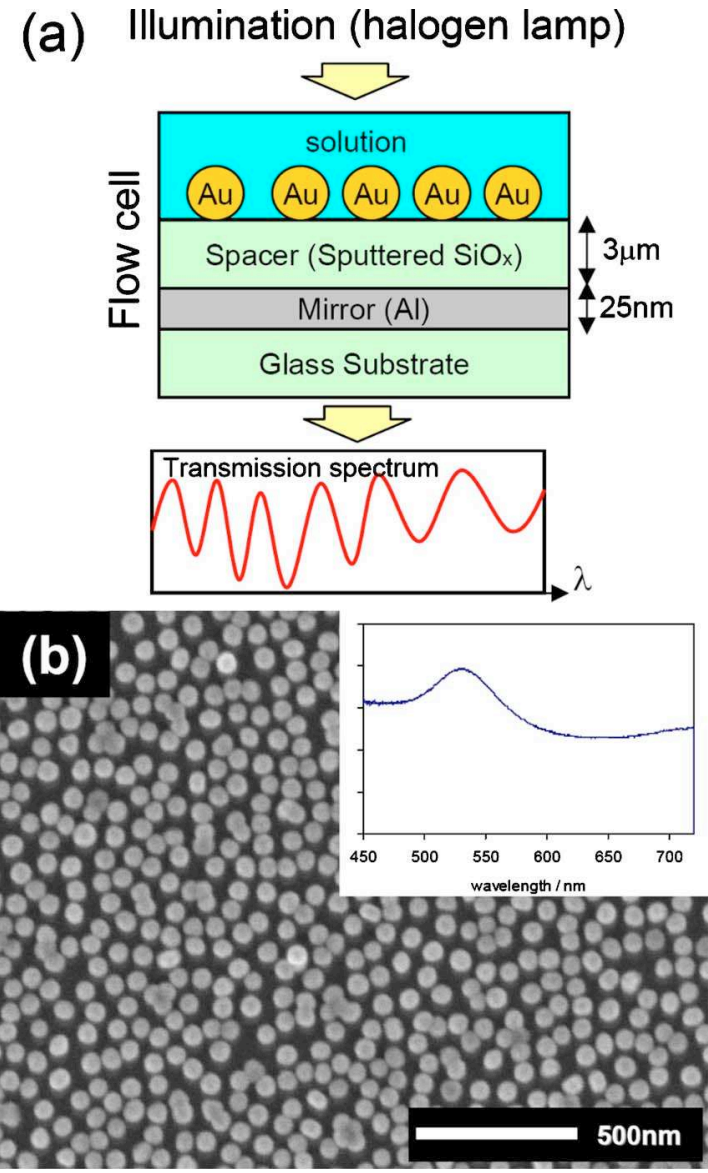

FIG. 1. (Color online) (a) Schematic drawing of the experimental setup for the transmission interferometric plasmonic sensor. (b) Scanning electron micrograph of $50 \mathrm{~nm}$ gold particles immobilized on a TInAS sensor chip. In the inset of panel $b$ the extinction spectrum of such gold nanoparticles without mirror layer is shown.

surements was $100 \mu \mathrm{g} / \mathrm{ml}$. The bulk sensitivity was measured by two different media: water $(n=1.333)$ and isopropanol $(n=1.3772)$.

Transmission spectra were recorded by an Ocean Optics spectrometer using halogen lamp illumination and simple fiber optic readout. In this setup only the perpendicularly transmitted light is collected. The acquired spectra were normalized by the spectrum of the reference substrate with only aluminum mirror layer coating. The schematic drawing of the experimental setup and a scanning transmission electron micrograph of a Au-particle coated sensor are shown in Fig. 1.

\section{B. Signal processing}

We used two different evaluation methods to process the interference spectra. In the first method the peak and dip intensities of the raw spectrum were detected and the ratio was defined as the signal

$$
\operatorname{PDR}\left(\lambda_{\text {avg }}\right)=\frac{I\left(\lambda_{\text {peak }}\right)}{I\left(\lambda_{\text {dip }}\right)},
$$

where $\lambda_{\text {avg }}=\left(\lambda_{\text {peak }}+\lambda_{\text {dip }}\right) / 2$. Note that this peak dip ratio (PDR) is a self-referenced intensity-based signal as the intensity fluctuation of the illumination is automatically compensated. Always adjacent peaks and dips were used for 
(a)

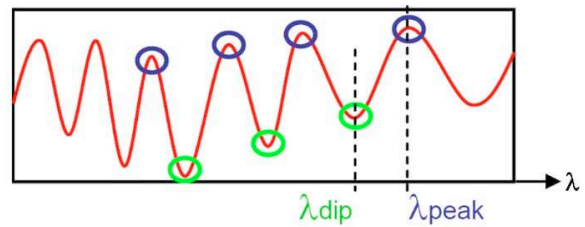

(b)

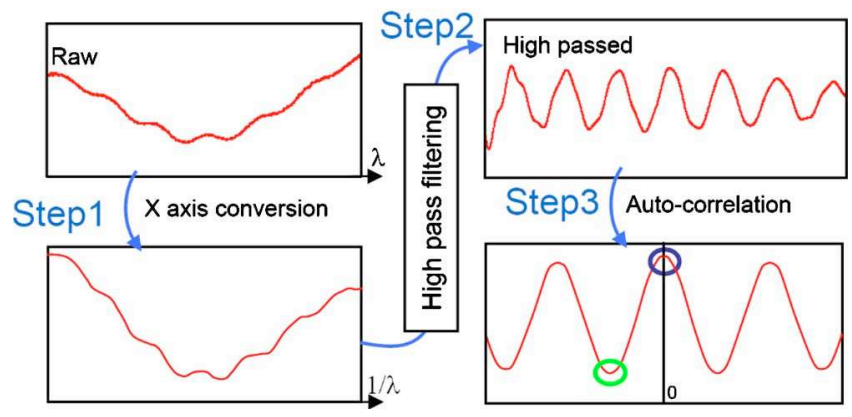

FIG. 2. (Color online) Schematic drawings of the (a) simple peak-dip detection (method 1) and (b) processing for the spectrum with small modulation (method 2). The detected peaks and dips are marked by blue (dark) and green (bright) circles, respectively. In the method 1, the peak/dip positions and intensities were determined from raw spectra. In the method 2, the spectrum was first reciprocally converted, followed by high pass filtering. The autocorrelation function of the processed spectrum was used to determined the peak and dip intensity.

PDR. When the wavelength of the PDR is indicated, it refers to the average of the peak and dip positions.

In the second method, we applied a series of mathematical transformations to the spectra. First the $x$ axis is converted from $\lambda$ to $1 / \lambda$. This procedure makes the modulation quasiperiodic which is suited for the next Fourier filtering. Then, a high pass filtering was applied to extract only the periodic modulation of the interference from the spacer layer. Filtering is especially useful when the modulation is relatively small compared to the background which is mainly caused by absorption of the particles. It is important to include the zero order Fourier component after the high pass filtering process to maintain the self-referencing property. The processed data were further converted by taking its autocorrelation function. The first peak (at the origin) and dip were finally evaluated as the first method. The peak and dip in the autocorrelation function include information of all the peaks and dips which appears in the original function. These procedures are schematically illustrated in Fig. 2.

\section{Simulation}

The simulation was performed using the MAX-1 software package. ${ }^{14}$ For bulk sensitivity investigation the $50 \mathrm{~nm}$ gold particle was located $2 \mathrm{~nm}$ above the surface of the sensor substrate in order to avoid singularity [Fig. 3(a)]. We tested different gap sizes but no significant impact was observed. For the molecular adsorption simulation, the gap of $4 \mathrm{~nm}$, a $2 \mathrm{~nm}$ underlying molecular layer, and a molecular shell with the radius of $28 \mathrm{~nm}$ were modeled [Fig. 3(b)]. The TInAS substrate model consists of a $3 \mu \mathrm{m}$ spacer layer, a $25 \mathrm{~nm}$ aluminum mirror layer, and glass substrate underneath [Fig. 3(a)]. We used periodic boundary conditions with 100 and 80 $\mathrm{nm}$ for the lateral direction. In such conditions diffraction or (a)

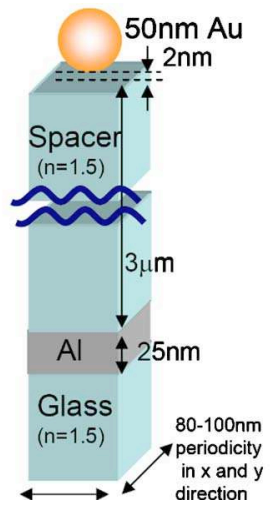

(b)

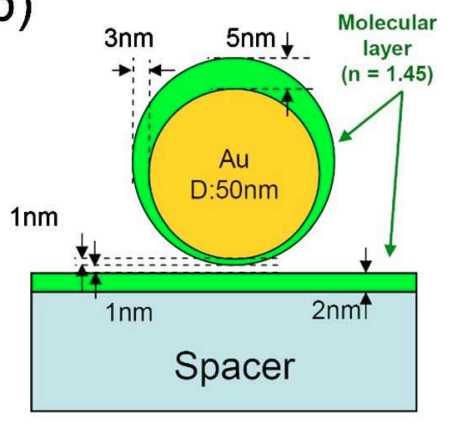

FIG. 3. (Color online) Schematic illustration of the simulated models of (a) the periodic unit cell and (b) the molecular adsorption layer. For the simulation without molecules (a), a $50 \mathrm{~nm}$ gold particle was placed at a distance of $2 \mathrm{~nm}$ from the substrate to avoid singularity. To simulate the molecular adsorption (b), the gold particle was located at a distance of $4 \mathrm{~nm}$ from the substrate to introduce molecular layers. The molecular layer on the particle is spherical with the diameter of $56 \mathrm{~nm}$ and with the $2 \mathrm{~nm}$ vertical displacement of the sphere center from the center of gold particle. For all the models, the thicknesses of the spacer and mirror layers were set to be $3 \mu \mathrm{m}$ and $25 \mathrm{~nm}$, respectively. The refractive index of 1.5 was used for the spacer layer and glass substrate, and 1.45 for the molecular layer.

interparticle coupling is negligible. The system was illuminated by a plane wave and the transmission spectrum was acquired as efficiency of the Rayleigh expansion located at the bottom of the simulated cell. ${ }^{14}$ The spectra were normalized by the simulated spectrum of the substrate with only an aluminum mirror layer to simulate the experimental normalization. Constant refractive indices were used for the dielectric materials, namely $1.333,1.45$, and 1.5 for water, adsorbed molecule, and spacer, respectively, since the dispersion of the dielectric constants for these materials is negligibly small (dispersion of $<1.4 \%$ within the wavelength range of $450-650 \mathrm{~nm}$ ) compared to metals. For the dispersive dielectric constant of the metals, measured data were used. ${ }^{15,16}$

\section{TIPS}

\section{A. Bulk sensitivity}

Figure 4 shows experimentally acquired TIPS spectra, simulated TInAS spectra (without plasmonic particles), and simulated TIPS spectra with different bulk media. The peak/ dip of the TIPS spectrum is roughly shifted by the phase $\pi$ from the TInAS spectrum as the plasmonic particle layer acts more like a mirror than a low refractive index medium. In TIPS the modulation of the oscillating pattern increases by higher refractive index media, while TInAS shows opposite behavior. This significant difference originates from the scattering property of the plasmonic particles. The backscattering power of gold nanoparticles typically increases in higher refractive index medium. On the other hand, the reflection at the medium-spacer interface in TInAS depends on the refractive index contrast which decreases with higher refractive index medium. Similarly by increasing the density of the nanoparticles [Fig. 4(d)], the modulation increases because the reflection of the top layer increases, thus increasing the finesse of the etalon. ${ }^{17}$ 

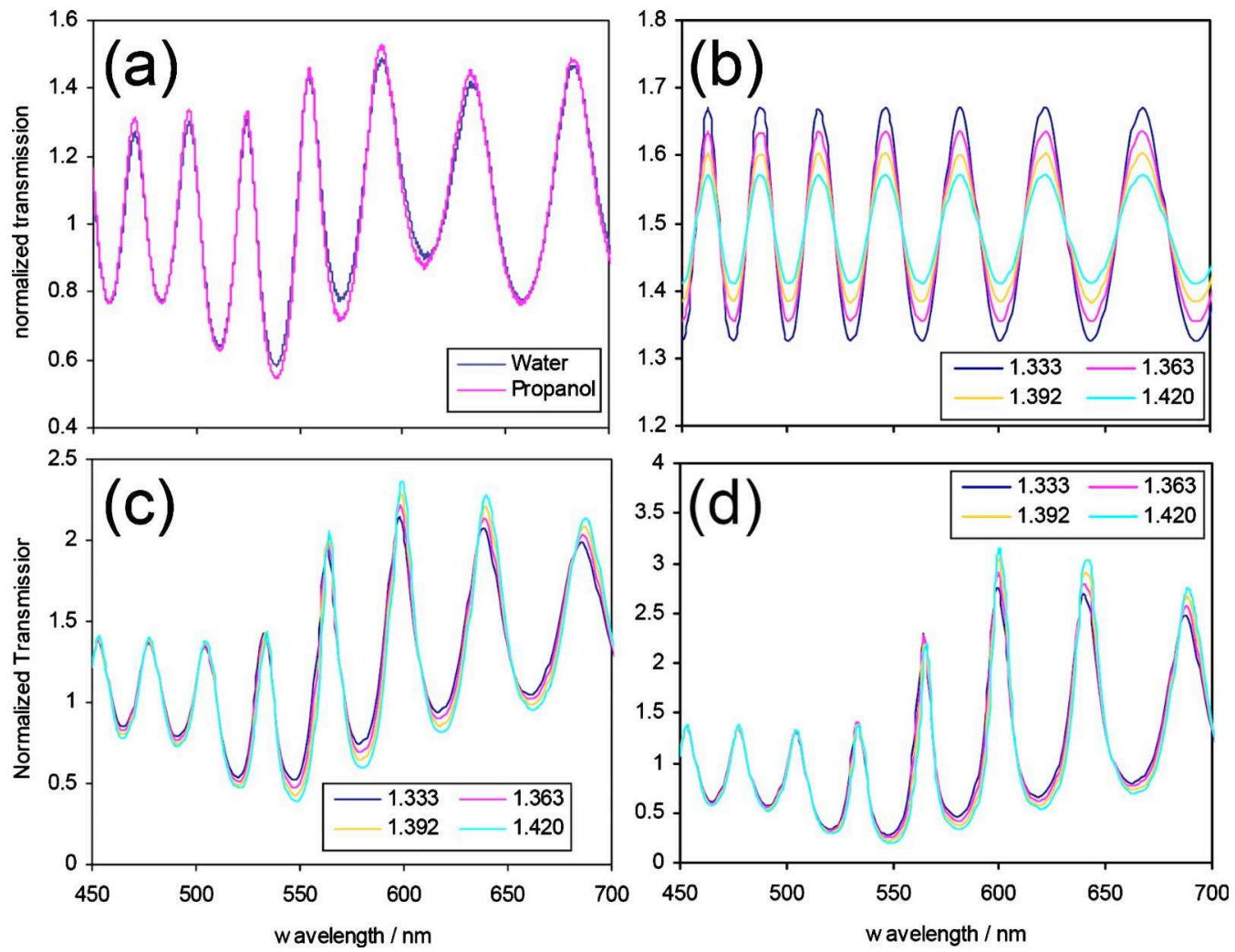

FIG. 4. (Color online) (a) Experimental TIPS spectra, (b) simulated TInAS spectra, and [(c) and (d)] simulated TIPS spectra with different bulk refractive index: water $(n=1.333)$ and propanol $(n=1.3772)$ for the experiment, and $n=1.333,1.363,1.392,1.42$ for the simulation. Periodicities of the simulated model [see Fig. 3(a)] was [(b) and (c)] $100 \mathrm{~nm}$ and (d) $80 \mathrm{~nm}$, which corresponds to the interparticle spacing of 50 and $30 \mathrm{~nm}$, respectively.

We define the bulk sensitivity as PDR signal per refractive index unit (RIU) of the medium. PDR/RIU data from experimental and simulated spectra with $100 \mathrm{~nm}$ period are plotted as a function of wavelength in Fig. 5. The highest signals are obtained around the resonance of the gold particles $(500-600 \mathrm{~nm})$ both in the experiment and in the simulation, which originates from the sensitive scattering of the gold nanoparticles. The absolute value of the experimental sensitivity is lower than in the simulation, which can be explained by the limited coherence of the wave due to the inhomogeneity of the particle size, shape, and distribution, and also the light source. The features seen around 530-560 $\mathrm{nm}$ both in the experiment and simulation originates from the dispersive background absorption spectrum of the gold par-

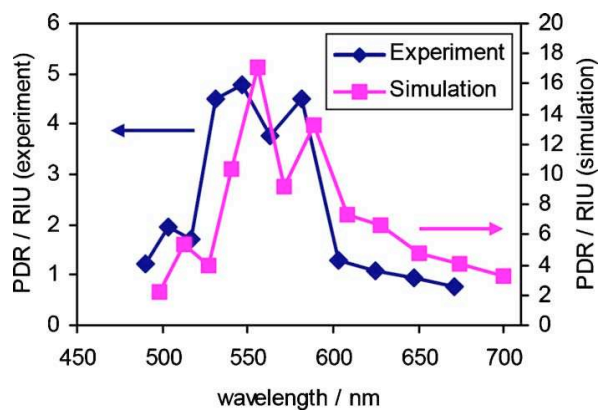

FIG. 5. (Color online) Bulk sensitivity calculated from the measured PDR and simulated PDR of the model with $100 \mathrm{~nm}$ period and $3 \mu \mathrm{m}$ spacer layer. The PDR was normalized by RIU. Each PDR is assigned the average peak/dip position and plotted as a function of wavelength. The experimental and simulated bulk sensitivities exhibit consistent wavelength dependence. ticles. The high absorption of gold particles at the interference peak position effectively results in a sensitivity dip around $560 \mathrm{~nm}$. Therefore, absorption can introduce nonuniform distribution of the sensitivity. Considering realistic noise, which is also discussed in the next section, a sensitivity of less than $2 \times 10^{-4}$ RIU can be readily achieved using a $0.43 \mathrm{~Hz}$ acquisition frequency. Although this sensitivity is a few orders of magnitude worse than the state-of-the-art methods at this acquisition rate, ${ }^{18}$ longer acquisition times and more averaging would further increase the $\mathrm{S} / \mathrm{N}$.

The sensitivity of TIPS does not depend on the quality factor (sharpness of the peak) of the plasmonic resonance, while most of the LSPR biosensing methods rely on the peak shift where a sharper peak gives better sensitivity. In principle TIPS accepts broad band resonances without a sharp distinct peak. This inherent tolerance signifies an advantage in the fabrication process because plasmonic resonances are typically very sensitive to small structural changes and often show broad resonances when fabricated. ${ }^{19}$ Since the sensitivity is dependent on the scattering of the plasmonic particles and the dipolar scattering is known proportional to the sixth power of the radius of the particle according to Mie's theory, use of larger plasmonic particles would improve the bulk sensitivity.

\section{B. Protein layer sensitivity for biosensing}

Protein layer adsorption measurements were conducted to test the practical sensitivity for biosensing. The experimental spectra before and after BSA adsorption (including 
(a)

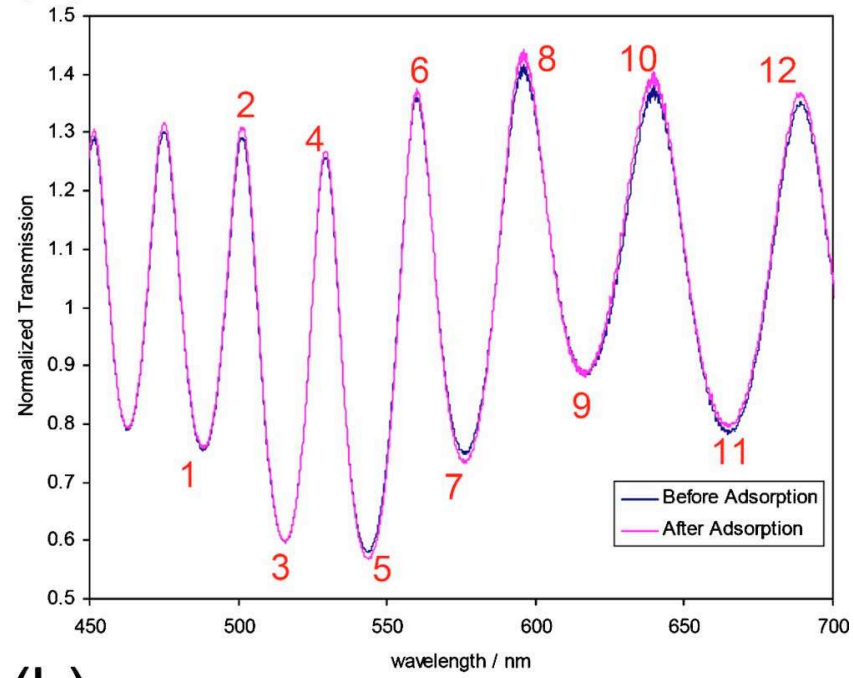

(b)

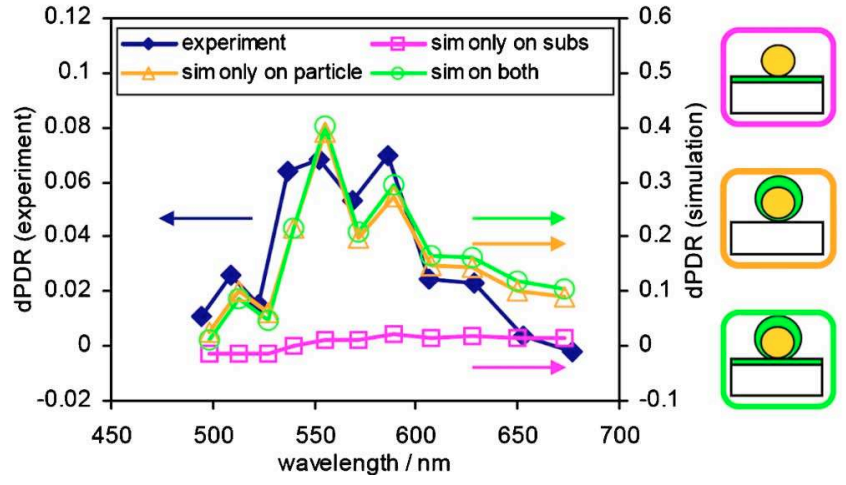

FIG. 6. (Color online) (a) Experimental normalized transmission spectra before and after BSA adsorption. The evaluated peaks and dips are numbered for the comparison of PDR signal. (b) PDR signal changes (dPDR) upon molecular adsorption of the experiment and simulation were plotted as functions of wavelength. Simulations were performed with different adsorption molecular layers: with a layer only on the substrate, with a layer only around the particle, and with layers both on the particle and on the substrate. Schematic illustrations are added with the same color scheme as the plotted lines in the inset.

rinsing with buffer) are shown in Fig. 6(a). Similar to the bulk medium change, the modulation amplitude increased after adsorption of BSA molecules. It is because the scattering of the gold particles increases when it is surrounded by a shell of higher refractive index material. The effect is the same as in the bulk case. The PDR signal change (dPDR) upon molecular adsorption is plotted in Fig. 6(b). The wavelength dependence is similar to the bulk sensitivity (c.f. Fig. 5). This adsorption measurement was conducted without specific functionalization of the gold particle or the substrate. In the simulation, we modeled different adsorption locations: (i) only on the substrate, (ii) only on the particle, and (iii) both on the particle and substrate. The thickness of the adsorbate in the simulation is in the order of the reported thicknesses of the BSA, which lie in the range of $3-5 \mathrm{~nm} .{ }^{20,21}$ The resulting PDR signal change (dPDR) is plotted in Fig. 6(b) together with the experiment. Situation (i) adsorption only on the substrate, did not exhibit apparent signal change (modulation intensity change) while (ii) the spectra with adsorption only
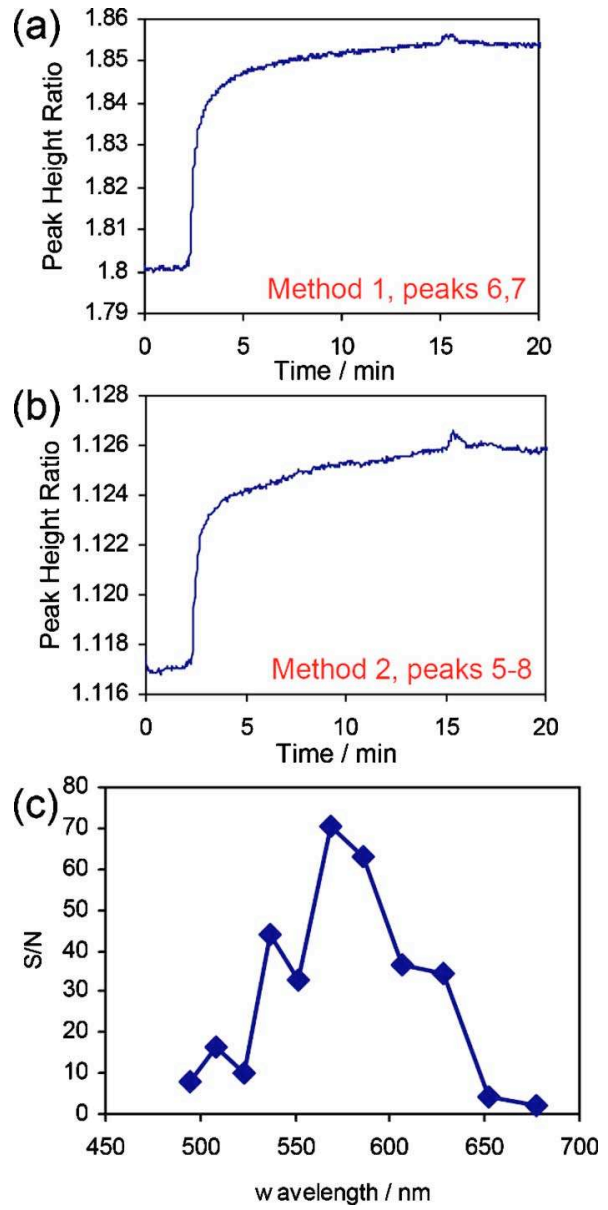

FIG. 7. (Color online) Adsorption kinetics of BSA (100 $\mu \mathrm{g} / \mathrm{ml}$ in HEPES) by (a) first evaluation method of taking PDR from the raw data and (b) second evaluation method including peaks/dips nos. 5-8 in Fig. 6. The peaks and dips used in panel a are no. 6 and no. 7 which are indicated in Fig. 6. BSA solution was injected at the time of $2.5 \mathrm{~min}$ and the flow cell was rinsed by HEPES buffer at $15.5 \mathrm{~min}$. (c) $\mathrm{S} / \mathrm{N}$ of the evaluated signals upon a BSA monolayer adsorption by method 1 [Fig. 2(a)]. The noise was estimated from the $3 \sigma$ of the baseline taken over $150 \mathrm{~s}$ with $0.43 \mathrm{~Hz}$ acquisition frequency. The average wavelength of the peak/dip position was assigned to each evaluation set and plotted as a function of wavelength. The $\mathrm{S} / \mathrm{N}$ of method 2 [Fig. 2(b)] was approximately 34 when peaks/dips nos. 5-8 were included.

on the particle, and (iii) on both exhibit roughly a similar amount of signal from the bare particle. This comparison also illustrates that the sensor is sensitive only to the adsorption on the gold particles. Therefore we can conclude that the spectral change we observed in Fig. 6(a) originates from the BSA adsorption on the gold particles.

Selected adsorption curves are shown in Figs. 7(a) and 7(b). We monitored different peaks and dips, which are numbered in Fig. 6(a). A signal increase was observed upon BSA adsorption and this signal level remained unchanged after rinsing, which confirmed that the change is due to the adsorbed layer and not caused by bulk refractive index change.

The S/N of the PDR upon BSA adsorption is summarized in Fig. 7(c). The noise level was taken as $3 \sigma$ of the baseline taken over $150 \mathrm{~s}$ with $0.43 \mathrm{~Hz}$ acquisition frequency. There is a good agreement between the measured $\mathrm{S} / \mathrm{N}$ and simulated wavelength dependence of BSA adsorption [Fig. 6(b)] and both follow the wavelength dependence 
of bulk refractive index sensitivity in Fig. 5. The slight redshift of the sensitive signal range in $\mathrm{S} / \mathrm{N}$ [Fig. 7(c)] compared to the bulk or layer sensitivity [Figs. 5 and 6(b)] can be explained by the spectral characteristics of the halogen lamp used here. Indeed, the spectrum of our lamp has a maximum around 570-600 nm, resulting in a peak maximum in S/N.

A data curve obtained by the second method (high-pass filtering + auto correlation) described in Fig. 2(b), which integrates the signal from the wavelength range of 535-605 $\mathrm{nm}$ (peak/dip nos. 5-8), is also shown in Fig. 7(b). These data did not show improved $\mathrm{S} / \mathrm{N}(\sim 35)$ compared to the best $\mathrm{S} / \mathrm{Ns}$ from the more direct first evaluation method. When all the peaks/dips nos. 1-12 were included, the S/N was decreased to 23 . This example shows that inclusion of only the sensitive wavelength range improves the $\mathrm{S} / \mathrm{N}$. The timeresolved limit of detection (LOD) estimated from the adsorbed mass of BSA (Ref. 21) is $\sim 5 \mathrm{ng} \mathrm{cm}^{-2} \mathrm{~Hz}^{-1 / 2}$, which is comparable to the TInAS system. ${ }^{3}$ Although state-of-theart biosensors, including high performance SPR and other interferometric methods, have higher sensitivity, $3,18,22$ proper choice of the spectrometer and optimization of the signal treatment could further improve the LOD of TIPS system. ${ }^{23}$

The advantage of the TIPS is the tunability of the two detection wavelengths. ${ }^{24}$ The position of the peaks and dips can readily be tuned by changing the spacer layer thickness, which simplifies fabrication over a precise plasmonic structuring.

\section{QUANTIFICATION OF DISPERSIVE COMPLEX REFLECTION COEFFICIENT OF PLASMONIC LAYER}

In this section we quantitatively analyze the TIPS spectra to extract the dispersive complex reflection coefficient of the plasmonic structure. The complex reflection coefficient can be defined as

$$
r=r_{0} \exp (i \Delta \phi),
$$

where $r_{0}$ is the amplitude and $\Delta \phi$ is the phase. In the following, we describe a methodology to extract the phase and amplitude information from one spectrum, which also includes the dispersive absorption of the plasmonic particles as background signal. This approach is also applicable for a TInAS system to measure the optical property of the adsorption layer. ${ }^{25}$ (The previously described TInAS measures only the phase shift.) The simulation results in this section were obtained from the model with $100 \mathrm{~nm}$ period [Fig. 3(a)].

\section{A. Phase determination}

First, we calculate the phase of the plasmonic scatterer from the spectrum measured in water. For the phase calculation we use the second data processing method [Fig. 2(b)] up to step 2 (axis reciprocation + low pass filtering) to extract the peak/dip positions with a minimum influence from the background by absorption. The positions of the peaks and dips of the interferometric pattern correspond to the constructive and destructive interference between the transmitted wave and the reflected wave, respectively, i.e., when the total phase shift $\left(\Delta \phi_{\text {total }}\right)$ composed by the mirror $\left(\Delta \phi_{\text {mirror }}\right)$, the spacer, and the plasmonic particles $\left(\Delta \phi_{\mathrm{AuNP}}\right)$ is $2 m \pi$ and $(2 m$ $+1) \pi$, respectively, where $m$ is an integer number,

$$
\Delta \phi_{\text {total }}(\lambda)=2 \pi\left(2 n_{\text {spacer }} L / \lambda\right)+\Delta \phi_{\text {mirror }}(\lambda)+\Delta \phi_{\text {AuNP }}(\lambda) .
$$

$n_{\text {spacer }}$ denotes the refractive index of the spacer layer, and $L$ the thickness of the spacer layer. As stated before, $\Delta \phi_{\text {total }}$ is $2 m \pi$ for the peak and $(2 m+1) \pi$ for the dip. The quantity $\Delta \phi_{\text {mirror }}$, which is dispersive due to aluminum, ${ }^{16}$ can be separately calculated from an one-dimensional layered model with a $25 \mathrm{~nm}$ aluminum layer interfaced by two semi-infinite glass $(n=1.5)$ media. $n_{\text {spacer }}$ and $L$ were set to be 1.5 and $3 \mu \mathrm{m}$ as in the previous simulations. We detected the peak and dip positions of both the experimental spectrum [i.e., the spectrum before adsorption in Fig. 6(a)] and the simulated spectrum with $100 \mathrm{~nm}$ period [see spectrum at $n=1.333$ in Fig. $4(\mathrm{c})]$ and calculated $\Delta \phi_{\text {AuNP }}$ from the position of the peaks and dips [Fig. 8(a)].

To confirm the validity of the calculated phase shift from the peak or dip of the spectrum, we calculated the phase shift of the plasmonic layer alone by simulation of a model without the mirror layer of Fig. 3(a) with an illumination from bottom. This simulated phase shift can be calculated at all wavelengths, and therefore plotted as continuous line (green) in Fig. 8(a), while the calculated phase shifts from the peaks and dips are discrete points at the peak/dip wavelengths. The calculated phase from the simulated spectrum (pink square dots) reveals a close match with the simulated green reference curve, while the phase of the experiment reveals quantitative and qualitative disagreement. The excess phase shift observed above $600 \mathrm{~nm}$ is probably due to the coupled mode or coagulated particles [see inset of Fig. 1(b)]. ${ }^{26}$ The difference of the phase shift in the experiment and simulation probably arises from the inhomogeneous density of the particles in the experiment, which results in the incoherent scattering compared to the perfect array in the simulation. Indeed, the simulated phase shifts were largely dependent on the spacing between the particles (data not shown). There may also be offset of the phase due to lower refractive index of the spacer layer or the different spacer thickness than the assumed values. (i.e., 1.5 for refractive index and $3 \mu \mathrm{m}$ for the thickness)

In the case of a single dipolar gold particle, the complex back scattering can be explicitly solved and it is proportional to the quantity $X$ which is expressed as ${ }^{10,11}$

$$
X=\frac{\varepsilon_{\text {gold }}-\varepsilon_{\text {medium }}}{\varepsilon_{\text {gold }}+2 \varepsilon_{\text {medium }}},
$$

where $\varepsilon_{\text {gold }}$ (Ref. 15) and $\varepsilon_{\text {medium }}$ are the dielectric constants of gold and surrounding medium. In Fig. 8(c) the phase of $X$ is plotted as blue line. The shape of the curve resembles the calculated and simulated phase in Fig. 8(a), but with a large offset. A single free particle cannot reflect the wave like a continuous metal plate. On the other hand the dense particle layer can act as a reflecting mirror by far field interaction, which results in the phase shift around $\pi$. This reveals that such a particle layer is not simply a sum of single particles but a metamaterial. 

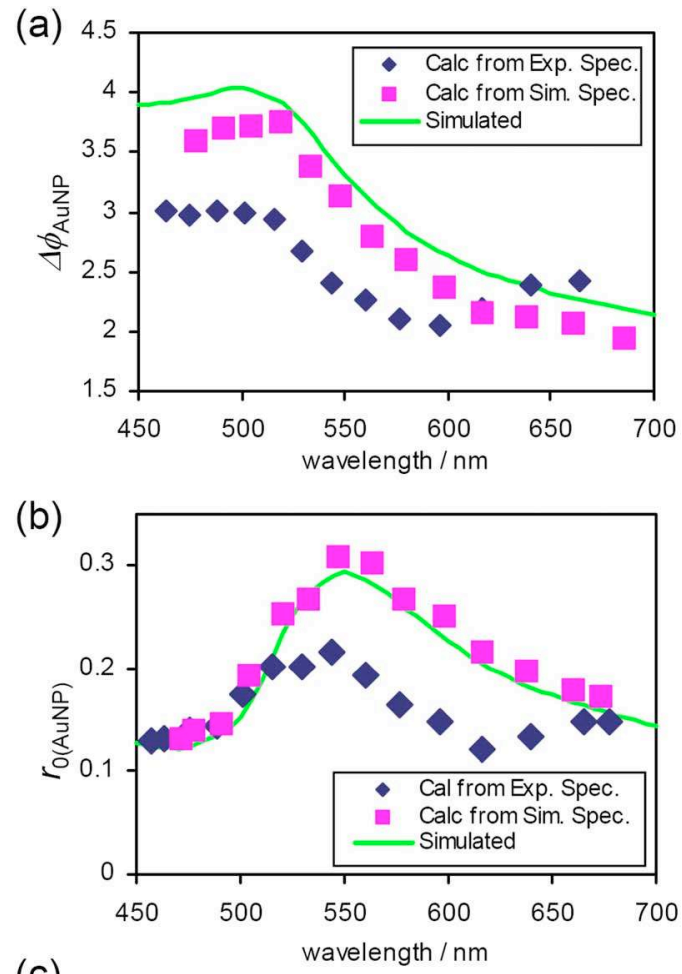

(c)

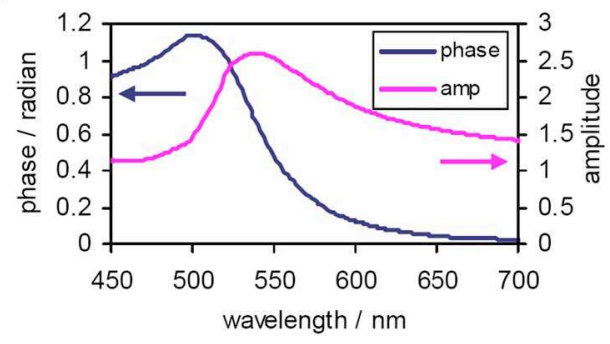

FIG. 8. (Color online) Complex optical reflection coefficient of [(a) and (b)] two-dimensionally dispersed plasmonic particles on a glass substrate and (c) a single dipole gold nanoparticle in free space. The calculated (a) phase shift and (b) amplitude of the reflection coefficient from the experimental and simulated spectra using Eqs. (3) and (5)-(9) are plotted as blue rhomboids (experiment) and pink squares (simulation), respectively. The simulated spectrum is of $100 \mathrm{~nm}$ period. The continuous green lines represent the (a) phase shift and (b) amplitude of the reflection coefficient directly acquired from the reflected electric field in the simulation model without the mirror layer, i.e., glass substrate with Au particles only. The plots of panel c are calculated from Eq. (4) and Ref. 13.

\section{B. Amplitude determination}

To calculate the amplitude of the reflection coefficient of the gold nanoparticle layer from the spectra, we define a new, absorption-independent, peak dip ratio ( $\mathrm{PDR}^{\prime}$ )

$$
\begin{aligned}
& \operatorname{PDR}^{\prime}\left(\lambda_{\text {peak }}\right)=\sqrt{\frac{I\left(\lambda_{\text {peak }}\right)^{2}}{I\left(\lambda_{\text {dip } 1}\right) \cdot I\left(\lambda_{\text {dip } 2}\right)}}, \\
& \operatorname{PDR}^{\prime}\left(\lambda_{\text {dip }}\right)=\sqrt{\frac{I\left(\lambda_{\text {peak } 1}\right) I\left(\lambda_{\text {peak } 2}\right)}{I\left(\lambda_{\text {dip }}\right)^{2}}} .
\end{aligned}
$$

For this we use two adjacent dips/peaks to normalize any intensity change by absorption. This new PDR' compensates the linear part of dispersive absorption in the background (absorption at the peak and absorption at the dip are different), and as such it is better suited for quantitative analysis of the absolute values. (Note that although PDR is robust to the change by multiplication, we had to use the raw spectrum data for the amplitude determination since Fourier filtering introduces a signal change by subtraction.)

The scattering amplitude is basically the amplitude of the reflection coefficient of the nanoparticle layer, which affects the shape of the spectrum of the etalon. The transmission spectrum of an etalon is known to be expressed as a function of phase $\phi$ (Ref. 17)

$$
I_{t}=\frac{1}{1+F \sin ^{2}\left(\frac{\phi}{2}\right)},
$$

where $F$ is the finesse coefficient. The finesse coefficient $F$ is defined as

$$
F=\frac{4 R}{(1-R)^{2}},
$$

where $R$ is a product of the reflection coefficient of the two facing reflecting interface of the etalon, namely $r_{0 \text { (mirror) }}$ and $r_{0(\mathrm{AuNP})}$

$$
R=r_{0(\text { mirror })} \cdot r_{0(\mathrm{AuNP})} \cdot
$$

It should be noted that the reflection coefficient $r_{0}$ here represents an amplitude in Eq. (2). Since PDR' is the ratio of the maximum and minimum intensities of an etalon, $\mathrm{PDR}^{\prime}$ can be expressed as

$$
\mathrm{PDR}^{\prime} \approx \frac{I_{\max }}{I_{\min }}=\frac{1}{1 /(1+F)}=1+F .
$$

The absorption factor due to the imaginary part of the dielectric constant is approximately cancelled out by taking the ratio. Since the reflection coefficient of the mirror layer can be calculated as previously, we can now determine the amplitude of the reflection coefficient (i.e., scattering amplitude) of the gold nanoparticle layer using these relationships. The amplitude of the reflection coefficient $r_{0}$, calculated from the experimental spectrum and the simulation spectrum with $100 \mathrm{~nm}$ period by using Eqs. (5)-(9) are plotted as discrete dots (experimental: blue rhomboid, simulation: pink square) in Fig. 8(b). As in the phase determination, the amplitude of the reflection coefficient $r_{0}$ directly determined from a simulation model without the mirror layer is plotted as smooth line. The simulated reference values are nicely reproduced by the calculation from the simulated spectrum, proving the validity of above procedure. A deviation of the experimental values is again observed and can be explained by the lower and inhomogeneous particle density and coupled (i.e., agglomerate) plasmon modes at the higher wavelength. The shape of the curve is reminiscent of the single dipole spectrum [Fig. 8(c), pink line] as suggested also by the phase measurement in the previous section.

\section{Phase and amplitude change by molecular adsorption}

The phase and amplitude changes in the reflection of the plasmonic particle layer upon molecular adsorption were calculated using the procedure described above. Figure 9 shows 

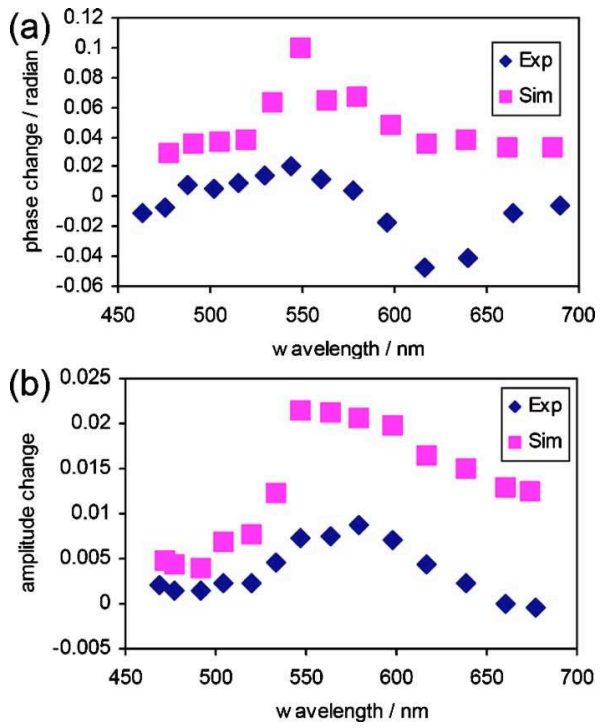

FIG. 9. (Color online) (a) Phase and (b) amplitude change in the gold particle layer upon adsorption of the molecule calculated from the experimental and simulated spectra. Experimental values are calculated from the BSA adsorption and the simulation values from the model with adsorption only on the particle as shown in Fig. 6(b)

the phase and amplitude changes by experimental BSA adsorption $(100 \mu \mathrm{g} / \mathrm{ml})$ and by simulated adlayer only around the particle [Fig. 6(b)]. The calculated phase change in Fig. 9(a) is quite different at the resonance of the plasmonic particles from off-resonance. These phase changes correspond to around $0.1 \mathrm{~nm}$ or smaller in wavelength. (The maximum value in the simulation corresponds to $\sim 0.5 \mathrm{~nm}$ ) Such small phase shifts by molecular adsorption are preferable because the monitored peak or dip would not move during the measurement where the fixed wavelength is practically used. Since the full width at half maximum of a typical light emitting diode light source is $\sim 25 \mathrm{~nm}$, which is much larger than the possible peak shift of $0.1 \mathrm{~nm}$, the peak/dip position would not shift away from the light source range when the light source peak is properly set on the peak or dip of the TIPS spectrum. In practical application for sensing, the calibration process would effectively compensate the effect of phase shifts.

The amplitude change by molecular adsorption in Fig. 9(b) shows strong increases in the wavelength range of 550$600 \mathrm{~nm}$, which agrees with the sensitivity dispersion in Fig. 6(b). This result confirms the back scattering (or reflection) increase in the plasmonic layer by molecular adsorption. The difference of the experimental results from the simulation is probably due to the coherence originating from the particle distribution. A further study, such as better controlled distribution of the particles in the experiment, would be needed for the full understanding of the change in complex reflection coefficient by adsorption of the molecule.

\section{CONCLUSION}

Refractive index determination and bio-sensing were demonstrated by a combinatory system of plasmonic particles and optical interferometry in transmission mode (TIPS). We have developed a signal evaluation method based on the transmission signal intensity that works with a minimum of two wavelengths, which can be customized by engineering the thickness of the spacing layer. The signal contains information about the change in the scattering power of the plasmonic particles, which has been rarely used in standard LSPR sensing. This origin of the signal was further confirmed by the analysis of complex reflection coefficient of the plasmonic particle layer, which can be readily calculated from a single transmission spectrum. A higher signal sensitivity was observed at the wavelength around the resonance of the plasmonic structure. While the sensitivity of conventional peak-shift-based plasmonic sensors depends on the sharp resonance peak (i.e., high slope), the presented method also works with a broad resonances. This makes the fabrication less stringent. The current performance of the sensor, including sensitivity, acquisition speed, etc., is not yet competitive with the state-of-the-art instruments but comparable to the previously described TInAS and conventional label-free biosensing methods ${ }^{3,18}$ with additional advantages in setup (only two wavelengths needed), stability (selfreferenced), tunability, and required fabrication tolerance of the plasmonic layer.

We have also developed a discretized method to extract the wavelength-dependent dispersive complex reflection coefficient of the adlayer on the etalon (spacer), and successfully applied it to a model TIPS system to extract the complex reflection coefficient of a plasmonic nanoparticle layer. The calculation from the simulated spectrum showed nice agreement with the separately simulated results, thus confirmed the validity of the methodology. The mechanism of TIPS signal, which depends on the reflection amplitude of the plasmonic particle layer, was also clarified by this approach. Here only one measured spectrum is required and no additional optical parts are necessary in a TInAS setup. In addition, the proposed method is more general and could also be used to fully characterize the effective optical constants of any adsorption layer in TInAS, which was a limitation until now.

\section{ACKNOWLEDGMENTS}

CCMX.

This study is financially supported by ETH Zürich and

${ }^{1}$ H. J. Trurnit and G. Bergold, Kolloid-Z. 100, 177 (1942).

${ }^{2}$ V. S. Lin, K. Motesharei, K. S. Dancil, M. J. Sailor, and M. R. Ghadiri, Science 278, 840 (1997).

${ }^{3}$ M. Heuberger and T. E. Balmer, J. Phys. D: Appl. Phys. 40, 7245 (2007).

${ }^{4}$ S. A. Maier and H. A. Atwater, J. Appl. Phys. 98, 011101 (2005).

${ }^{5}$ J. N. Anker, W. P. Hall, O. Lyandres, N. C. Shah, J. Zhao, and R. P. Van Duyne, Nat. Mater. 7, 442 (2008).

${ }^{6}$ D. Kim, K. Kerman, M. Saito, R. R. Sathuluri, T. Endo, S. Yamamura, Y. Kwon, and E. Tamiya, Anal. Chem. 79, 1855 (2007).

${ }^{7}$ D. Kim, K. Kerman, S. Yamamura, Y. Kwon, Y. Takamura, and E. Tamiya, Jpn. J. Appl. Phys. 47, 1351 (2008).

${ }^{8}$ H. M. Hiep, H. Yoshikawa, M. Saito, and E. Tamiya, ACS Nano 3, 446 (2009).

${ }^{9}$ Ch. Hafner, Phys. Status Solidi B 244, 3435 (2007).

${ }^{10}$ Y. Matsuo and K. Sasaki, Jpn. J. Appl. Phys., Part 1 40, 6143 (2001).

${ }^{11}$ P. Stoller, V. Jacobsen, and V. Sandoghdar, Opt. Lett. 31, 2474 (2006).

${ }^{12}$ T. E. Balmer and M. Heuberger, Rev. Sci. Instrum. 78, 093105 (2007). 
${ }^{13}$ T. Sannomiya, Ch. Hafner, and J. Voros, Opt. Lett. 34, 2009 (2009).

${ }^{14}$ Ch. Hafner, MaX-1: A Visual Electromagnetics Platform (Wiley, Chichester, 1998).

${ }^{15}$ P. Johnson and R. Christy, Phys. Rev. B 6, 4370 (1972).

${ }^{16}$ D. R. Lide, CRC Handbook of Chemistry and Physics, 89th ed. (CRC, Boca Raton, 2008).

${ }^{17}$ M. Born and E. Wolf, Principles of Optics, 6th ed. (Pergamon, Oxford, 1980), pp. 323-364.

${ }^{18}$ X. Fan, I. M. White, S. I. Shopova, H. Zhu, J. D. Suter, and Y. Sun, Anal. Chim. Acta 620, 8 (2008).

${ }^{19}$ T. Sannomiya, P. K. Sahoo, D. I. Mahcicek, H. H. Solak, Ch. Hafner, D. Grieshaber, and J. Vörös, Small 5, 1889 (2009).
${ }^{20}$ T. J. Su, J. R. Lu, R. K. Thomas, Z. F. Cui, and J. Penfold, J. Phys. Chem. B 102, 8100 (1998).

${ }^{21}$ J. H. Teichroeb, J. A. Forresta, and L. W. Jones, Eur. Phys. J. E 26, 411 (2008).

${ }^{22}$ X. D. Hoa, A. G. Kirk, and M. Tabrizian, Biosens. Bioelectron. 23, 151 (2007).

${ }^{23}$ A. B. Dahlin, J. O. Tegenfeldt, and F. Hook, Anal. Chem. 78, 4416 (2006).

${ }^{24}$ P. Neuzil and J. Reboud, Anal. Chem. 80, 6100 (2008).

${ }^{25}$ A. R. G. Alig, D. Gourdon, and J. Israelachvili, J. Phys. Chem. B 111, 95 (2007).

${ }^{26}$ M. Quinten, Appl. Phys. B: Lasers Opt. 73, 317 (2001). 